\title{
Cognitive behavioural therapy training in Tanzania: a qualitative study of clinicians' experiences
}

\author{
Lydia Stone ${ }^{1, *}$ and Megan A. Arroll ${ }^{2}$ \\ ${ }^{1}$ ATTACH Team, Oxfordshire County Council Children's Services, 4440 Nash Court, John Smith Drive, Oxford Business \\ Park South, Oxford OX4 2RU, UK and ${ }^{2}$ Simply Research, Suite 33, 10 Barley Mow Passage, London W4 4PH, UK \\ *Corresponding author. Email: lydia.stone@oxfordshire.gov.uk
}

(Received 09 March 2018; revised 07 January 2019; accepted 11 January 2019)

\begin{abstract}
Cognitive behaviour therapy (CBT) in an effective technique used widely in Western countries. However, there is limited evidence as to the utility, perception and understanding of CBT in developing countries. This study describes and investigates the experience of CBT training and practice in a group of practitioners in Tanzania. A qualitative approach to the investigation was used, and seven participants from Tanzania's only psychiatric hospital who had completed CBT training were interviewed. The resulting verbatim transcripts were analysed via interpretative phenomenological analysis. Five superordinate themes emerged from the analysis: 'the medical model', 'novelty', 'practicalities', 'process' and 'therapist effects'. The two themes 'therapist effects' and 'process' are discussed in detail as these may be particularly relevant to the clinical application of the therapy in a new culture and context. The implications for future development of CBT in Tanzania are considered. In developing CBT for dissemination in Tanzania, specific cultural and clinical issues highlighted by indigenous clinicians need consideration.
\end{abstract}

\section{Key learning aims}

(1) To identify what range of factors may influence clinicians' perception of and learning about CBT when CBT training is delivered in a mental health setting in Tanzania, or to those with East African origins.

(2) To consider the use of qualitative research approaches to inform the early stages of adapting CBT for use in new settings and applications.

(3) To be able to apply knowledge about cultural differences to standard CBT in order to promote culturally sensitive practice.

Keywords: cognitive behaviour therapy; cultural adaptation; training; qualitative methods

\section{Introduction}

Cognitive behaviour therapy (CBT) refers to a range of talking therapies, which are based on cognitive and behavioural theories of psychology, and which research has shown are effective in treating a wide range of emotional or psychiatric disorders (Grazebrook and Garland, 2005). Empirical evidence for its use in various patient groups has been accumulating over recent years, but the majority of research studies have been on populations in Western countries (Roth and Fonagy, 2005).

Data on rates of mental health problems in non-Western countries, particularly low and middle income countries (LAMIC henceforth), is often sparse, but literature indicates that there are likely to be equivalent rates of such problems. Certain unique factors may raise the prevalence of some 
difficulties, such as the socio-psychiatric issues that are comorbid with the HIV epidemic in sub-saharan Africa (Bolton and Wilk, 2004). Worldwide, clinicians see a treatment gap for mental health problems, but it is widest in LAMIC, with various factors being thought to hinder treatment provision, such as under-investment in mental health services and neglect/abuse of human rights of people with mental illness (Saxena et al., 2007). Some examples of mental health initiatives such as PRIME (Programme for Improving Mental Healthcare - focusing on improving the coverage of treatment in five LAMIC with support from global partners) and the Friendship Bench (a Zimbabwean project using lay health-workers to deliver problem-solving talking therapy) indicate the enthusiasm being generated regarding mental health worldwide and in Africa (see http://www.prime.uct.ac.za/prime-about-us and https://www.friendshipbenchzimbabwe.org/). These examples indicate that CBT interventions appear to enjoy some favour for adaptation and testing in LAMICs, due to their proven evidence base and highly structured nature, which makes training and dissemination relatively easy and cost-effective, compared with other psychotherapeutic interventions.

As the interest in global mental health continues to grow, together with the evidence base of effective interventions to treat people with mental illnesses in LAMIC, the focus has now turned to the need to provide 'action-able' and sustained training to staff (Thornicroft and Semrau, 2018). The EU-funded Emerald (Emerging mental health systems in low- and middle-income countries; see www.emerald-project.eu) programme, which is investigating mental health system strengthening in six LAMICs, aims to look at barriers and solutions to this (Semrau et al., 2015). Our interest in these issues in Tanzania led us to want to evaluate CBT training through discovering clinicians' personal reflections on such barriers and solutions in the Tanzanian context.

Previous research has identified the usefulness of integrating mental health care into primary care, as well as acknowledging Tanzania's commitment to mental health through its policies, budgets and training activities (Mbatia and Jenkins, 2010). In 2008, an amendment to the Tanzania Mental Health Act emphasized access to quality services and the rights of the mentally ill, as well as measures to ensure family and community involvement. The Mental Health Global Action Plan (mhGAP), launched by the WHO in 2013, continues to look at capacity building, universal health coverage, human rights and evidence-based practice and most recently has focused on an initiative to develop a psychological intervention workforce development package (WHO, 2017). A free app and training manuals provide non-specialized healthcare providers with information to help them diagnose and treat a range of mental, neurological and substance use (MNS) disorders. An evaluation of the potential CBT could offer as part of such psychological interventions seemed timely.

Furthermore, CBT could be of particular use in Tanzania, given its strong empirical evidence for use in those patient groups that exist in Tanzania. Marwick and Kaaya (2010) cite figures for depression in vulnerable populations in Tanzania from 15.5\% (HIV-positive patients) to 45\% (post-partum mothers) and Ngoma et al. (2003) report rates of common mental health disorders (such as depression and anxiety disorders) between 24 and $48 \%$ in patients attending primary health or traditional healer clinics in Tanzania. Literature looking at the use of CBT in LAMIC is still growing, but studies have shown its successful application in sub-saharan Africa, e.g. to reduce risky sexual behaviour amongst HIV+ patients in Zambia (Jones et al., 2005), reduce alcohol use among HIV+ outpatients in Kenya (Papas et al., 2010) and to improve mood in anxious Nigerian surgical patients (Osinowo et al., 2004).

No studies examining the use of CBT in Tanzania could be found by the authors. However, a review of psychological therapy evaluations in Pakistan and Uganda (both rural community settings) and a mixed rural-urban setting in India found that talking therapy resulted in significantly higher recovery rates from depression and anxiety, in comparison with usual care (Patel et al., 2011). Pertinently, the authors of this study noted that there was a human resource barrier to providing psychological treatment, which was overcome by the use of lay health workers with relatively brief training periods (the Pakistani programme consisted of only three days instruction in total). In additional to this practical issue of staffing, Chowdhary et al. (2014) suggest that cultural 
and contextual characteristics such as language (for example, using metaphors to increase cultural relevance, which also allows abstract concepts to be expressed concretely, simply and therefore more understandably) and social dynamics should be considered carefully when adapting psychological therapies for LAMIC. Nevertheless, research in developing countries has shown that accessible, affordable and culturally sensitive community and primary care psychological treatments that are evidence-based such as CBT are effective management tools for mental health disorders (Patel, 2007). Although talking therapies may be a useful addition to the treatments available to patients, other writers argue that psychotherapy is of little relevance in Africa due to different sociocultural and religious understandings of physical and mental illness (e.g. Aina, 2006).

Taking the findings of this literature into account, we were interested in the views of local clinicians who were familiar with these issues in their day-to-day interactions with and treatment of Tanzanian psychiatric patients. Quantitative measures were initially used, and these results are published elsewhere (Stone and Warren, 2011). However, we felt a qualitative approach could enhance our understanding of this topic as these methods access a broader range of opinions, unrestricted by quantitative measures. Furthermore, such qualitative studies are often thought to be helpful in the process of developing new programmes or services (Stewart et al., 2007). Therefore, this paper looks at the views of Tanzanian clinicians working in Mirembe National Psychiatric Hospital on CBT following a short training course in the principles and application of this therapeutic technique by one of the authors (L.S.). In order to develop culturally sensitive therapeutic treatments, the views of connected people (whether therapists delivering the therapy, or those receiving it) are essential. Such projects have been started elsewhere, such as in Pakistan (Naeem et al., 2010, 2016). These authors found specific thematic issues arising concerning the adaptation of CBT for local effectiveness and the obstacles felt to hinder its use, such as service issues, dealing with somatic complaints, the status of psychotherapy alongside medication, CBT homework, expectations about mental health services and illness beliefs. We therefore believe this type of exploration is necessary to consider when developing treatment systems, as well as ensuring that culturally sensitive practice is valid.

\section{Method}

\section{Context and setting}

Tanzania is a country in East Africa with a population of approximately 43 million. The African population consists of over 120 ethnic groups, with smaller communities of people of Arab, Indian and Pakistani origin. Whilst Swahili and English are the official languages, most Tanzanians know their tribal language, Swahili and various degrees of English, depending on their level of formal education. Most Tanzanians would seek help for mental health problems from a traditional healer or primary health clinic (Ngoma et al., 2003); only one specialist psychiatric hospital exists and is often used at crisis points when all other alternatives have been exhausted.

Mirembe Hospital, situated in Tanzania's capital city of Dodoma, is Tanzania's only psychiatric referral hospital. The majority of health workers at Mirembe are Nurse Attendants with limited basic training; professionally trained staff are relatively few, such as Enrolled and Psychiatric Nurses, Clinical Officers and Medical Doctors. There was only one fully trained psychiatrist at the time of this study. Approximately 100 clinical staff work in the hospital, which has an inpatient population of up to 540 as well as serving outpatients, who attend for both physical and mental health reasons.

\section{Sample}

The Cognitive Behaviour Therapy in Tanzania (CBT-Tz) Programme was developed by the author (L.S.), who at the time of the training had 5 years post-qualification experience using $\mathrm{CBT}$ as her main approach and receiving supervision from therapists recognized by the 
Table 1. Characteristics of interviewees

\begin{tabular}{lll}
\hline Participant & Profession & Gender \\
\hline Amani & Clinical officer & Male \\
Fred & Social worker & Male \\
Gervas & Senior mental health nurse & Male \\
Joseph & Consultant psychiatrist & Male \\
Julius & Assistant medical officer & Male \\
Pamela & Assistant medical officer & Female \\
Violet & Senior mental health nurse & Female \\
\hline
\end{tabular}

British Association of Behavioural and Cognitive Psychotherapy. The programme of sessions aimed to develop the skills of the trainees so that they could conduct sessions with patients (primarily with Axis 1 disorders) using CBT. Two courses of the CBT-Tz programme were carried out using the same material, but in slightly different formats: a course of twelve 3-hour workshops over a period of 4 months, and an intensive course of 3 days. The workshops involved didactic teaching, individual and group exercises, and homework tasks. Entry to the CBT training programmes was open to all staff at Mirembe hospital and also other interested staff working in mental health settings elsewhere.

Nineteen members of staff completed the training. During and after the course each participant was encouraged to apply CBT to their own patients, join the trainer in cases in a CBT clinic and attend supervision. They did this to varying degrees, in accordance with their existing work pressures and geographical proximity to Mirembe Hospital (seven of the participants of the second course did not work at Mirembe). Seven participants (two women and five men) agreed to be interviewed, all of which had basic training in counselling skills prior to the CBT programme and at least 2 years experience working at Mirembe. Their current roles within the organization ranged from social worker to consultant psychiatrist (Table 1).

\section{Procedure}

Three months after completion of the second course, each participant (of both courses) was sent a letter inviting them to discuss their experience of the training and CBT in general with the trainer (L.S.). The letter included an information sheet with details about the interview, which outlined important ethical considerations such as confidentiality (of both the interview itself and subsequent data) and anonymity (achieved by the use of pseudonym and only collecting background information pertinent to the study). Furthermore, due to the power dynamics, both organizational and cultural, a statement noted that negative consequences would under no circumstances result from either non-participation or withdrawal from the study. Informed consent was achieved via a consent form, included in the information pack, again to limit undue perceived pressure to participate. Once consent forms were received, the trainer contacted the participants, either by telephone or in person, to arrange a suitable time and place for the interview. Five interviews took place at Mirembe, whilst two participants preferred for the trainer to conduct the interview in their workplace. Interviews were recorded and immediately transferred to a laptop which was securely stored.

A semi-structured interview schedule was used to elicit responses. This consisted of a welcome/ introduction followed by three main sections of questions about clinicians' experiences of and views about CBT. A funnelling approach was used (Guba and Lincoln, 1981), so that a general question was asked ('please tell me about your experience of CBT, to start with your training'), followed by other more specific questions (e.g. 'did you have any understanding of what CBT itself was before training?') to achieve a depth of response from the interviewee. The interviews lasted between 28 and 61 minutes, with the average being 40.7 minutes $(S D=10.2)$. Interviews were 
conducted in English. The possibility of linguistic misunderstandings was considered due to the manner in which Swahili and English are used in Tanzania (which L.S. was familiar with, having worked there for 18 months); however, it is hoped that the range of verbatim quotations in the findings section below reflects the lived experience of the participants' narratives.

\section{Analysis}

Interpretative phenomenological analysis (IPA) is a qualitative tool that aims to demonstrate the rich and detailed personal experience of individuals by positioning lived experience at the centre of analysis rather than constructing accounts; hence the primary focus of such analysis is within the context of wider macro-level social and cultural factors (Smith, 2011; Smith et al., 2009). IPA is phenomenological as it involves the detailed and precise examination of lived experience as being 'real' and valid to the individual, as such each case within the body of data is analysed in detail and with the same merit as every additional case. IPA also offers a double hermeneutic process in that the participant is making meaning of their experience in their account to the researcher, but the researcher is also making sense of the participant's accounts which results in an interaction between the researcher and the data.

It was felt that IPA would be appropriate for the present study as the aim was to gain insight into the experience of hospital staff in Tanzania who were using a novel clinical approach, namely CBT. Furthermore, as is common in research in LAMIC, limitations in resources meant the trainer also took the role of interviewer and so it was important to consider how this might influence the data. As previously stated, IPA requires the researchers' interpretations of the narratives in order to enrich the accounts and qualify recommendations made. We aimed to adhere strictly to the guidelines from Smith et al. (2007) regarding the process and presentation of IPA work.

In terms of the process of IPA in this study, first a transcript was read thoroughly and initial descriptions, interpretations and themes were noted. This was then carried out for each subsequent transcript, bearing in mind previous findings whilst also keeping an open mind to additional descriptors. When all cases were analysed, attempts were made to identify patterns and thematic connections and superordinate themes were produced from a consolidated list of individual themes (Smith and Osborn, 2003). Transcripts were re-read to ensure that the findings were indeed representative of the narratives. The final step linked transcript quotations to each theme. In the present study, the first two stages of this process were done by each researcher separately and the final stage of developing the list of themes, deciding on superordinate themes and choosing verbatim quotations was achieved in face-to-face meetings jointly.

\section{Findings}

An open and interested approach to CBT was evident in participants' narratives of their experience of the training. In our analysis, five main themes emerged as contributing to the clinicians' experiences of CBT. There were lower-order themes within the main categories (see Table 2). The themes that emerged are described and illustrated with quotations below.

\section{Medical model}

The first theme labelled 'Medical model' includes three subordinate areas, as described in Table 2. The use of a non-pharmacological treatment appeared striking to the clinicians and was clearly stated by Amani and Gervas:

'CBT is err ... is a psychotherapy, talking with the patient without any medication.' (Amani)

'... because CBT is a treatment without medication' (Gervas) 
Table 2. Master and subordinate themes

\begin{tabular}{ll}
\hline Master themes & Subordinate themes \\
\hline Medical model & Not pharmacological \\
& Body/mind distinction \\
& Different selection of treatments for patients \\
& CBT is hidden \\
& Training is needed \\
Novelty & Attitudes of others \\
& Empowerment/patient focus \\
Process & Collaboration \\
& Context/scope \\
& Communicating with the patient \\
& Helping the therapist \\
Therapist effects & Self-disclosure/boundaries \\
& Understanding the model \\
& Beginner status \\
& Time factors \\
Practicalities & Facilities \\
\hline
\end{tabular}

The interviewees were accustomed to offering primarily or combined drug-based interventions for mental health issues before the introduction of the CBT training programme. This reliance on a very orthodox model of health and illness was also perceived to be held by patients, as demonstrated in Fred's narrative:

'People are not used to come and just talk, especially when they come to the hospital, they want to get treatment. He is there, he feels like he is sick, he want to be checked in the laboratory and he want to be told that, hey, you have malaria, that's why you have been feeling like this. But one, once you tell this person that this is nothing to do with physical illness, well for some people they don't agree with that one. And when you tell them to go to CBT, what is CBT? Yeah, you are going to have sessions of six weeks, once every week ...' (Fred)

As Fred points out, his patients sought a physical diagnosis for their symptoms to help them understand their condition. There appeared to be a lack of recognition that psychological processes could contribute to symptomatology. This led to the need for some additional explanations that may not have been required in Western countries as there is greater acceptance that emotional difficulties can lead to a myriad of symptoms including heart palpitations and dizziness in anxiety disorders and fatigue and sleep disruption in depression.

This difficulty in moving away from pharmacological interventions may have been due to the view that the mind and body are separate, i.e. a dualistic model of mental health was held. As Amani notes, he previously felt compelled to prescribe medication, even though there were clear signs of psychological distress in his interaction with the patient.

'Yes, you have to give medication. But some time patient came with, err come with headache, talking the detail from home, I mean the situation of the life, trying to check malaria whatever, it's negative and always complaining headache, sometimes loneliness ... so you know, err, this patient, you have to offer CBT.' (Amani)

The addition of CBT as a treatment option for patients did not come without resistance; as Joseph reports, the link between a psychological condition and a psychological treatment had not yet been made in his patient group. 
'If you have malaria, you believe in Chloroquine, you believe on anti-malarial, then you get cured. If you have another kind of fever, you believe on medication, you go for that, you get cured. But if you don't believe on the talk, you think that it's going to be rather few in the therapy, it's a doubt ...' (Joseph)

The insistence that patients need to 'believe' in talking therapy appears very astute, because individuals need to be committed to playing an active role in their CBT treatment, otherwise results can be poor. This, again, maybe be an important consideration in tailoring CBT to a non-Western audience, in the sense that there may need to be a supplementary and preliminary stage whereby some work is done to explain to the patient that there is this association between their symptoms, psychological or emotional distress and psychological therapy.

\section{Novelty}

The second overarching theme found in the data labelled 'Novelty' concerns views on this emerging approach. In terms of the novel nature of CBT and the lack of awareness of this technique, Joseph insists that a process of knowledge building would be important to encourage patients to acclimatize to this new approach:

'Because, look here, because of the, CBT is something like very new here. Because it is new, people know little about it. And because of that, then less people are, have that willing, have that interest in it, so the first thing may be to build interest to people.' (Joseph)

Joseph clearly states that without knowledge of CBT, individuals would be less willing to engage with the therapy. It is also apparent in Pamela's narrative that this process of familiarization would be both key to clinicians as well as patients.

'It was a new thing to experience and, err, I was bit hesitating if the, the new approach of therapy, err can be familiar to our clinical setting. Saying that, being Tanzanian, it was not something was familiar.' (Pamela)

However, this is not to say that the clinicians were negative towards this new tool; in fact, there was a consensus in the group that even though CBT was not yet in the public domain, a different type of therapy (i.e. non-pharmacological) was in demand.

'It means that it was a therapy which was hidden, but it was needed.' (Pamela)

This new technique was not perceived to come without difficulties, however. All seven participants noted that training was needed, as highlighted by Violet.

'I know, even now, at the school level, health school or colleges, CBT is not there. It's not included in the curriculum. So if I can suggest or give advice that if possible may be the Ministry of Health could add the CBT because it's very helpful.' (Violet)

Linked to training and patient awareness of this novel treatment approach, is the sub-theme of the attitudes of others. The participants actively encouraged others to try the techniques, as evidenced in Violet's account.

'And I, I even taught to my co-workers, to, to, to try to do CBT for their own family, for their future plan. Err, there were, there were some people who came here to ask me how to go with 
it, and some were client, they have got diabetes, and one have got infection with HIV, but from CBT they came here to ask how to go with it and how to plan the life.' (Violet)

Violet could clearly see the further applications of CBT outside of mental health conditions in the form of helping an individual cope with a chronic illness such as diabetes or HIV. This 'snowballing' effect demonstrates the importance of attitudes and the open-mindedness to participate in a new psychological skill. In fact, Julius notes that colleagues also believed in the progressive quality of this novel approach and hence demonstrates the power and influence of attitudes on others.

'Most of the people whom I've seen having therapy from comrades, from you, from me, that one I told you, have been very positive after the therapy. And I think that's the, the, the most, the strongest point of this therapy.' (Julius)

In addition to peers and colleagues, and within a culture where family relationships and hierarchies are very prominent, the impact of parents' attitudes was often cited by the participants as a significant factor in whether a patient will be open to CBT, as Joseph illustrates.

'Parents initiate CBT more than the patient. You can hardly ever find a patient asking for CBT. You can get one or two, you can get nine parents who knows about CBT.' (Joseph)

The influence of parents and families towards the commencement of this type of intervention could also be negative, i.e. patients with family members who were more wary of such a novel technique were less likely to try CBT. This sense of mistrust that CBT alone would prove effective was also perceived to come from higher management; therefore not all attitudes were positive.

'... For sure, the therapy is not considered potential as it was supposed to. Because even my seniors, they can just say, "Okay. If you have a CBT clinic but do you think, err, you can't do both? Go to the ward and the go to see the patient?" But I tell them, "No, I have a good number of them", they said, "Ah, what are you doing there? Oh, what are you doing there?" So ..." (Pamela)

\section{Process}

The clinicians described several unique features they experienced in the course of learning and using CBT which can be seen to all relate to the theme of the 'Process' through which CBT occurs. 'Process' is often distinguished from 'content' in psychotherapy, to distinguish what may have been learnt or said (content) from how it was learnt/said (process). It is interesting that this arose as a theme because awareness of process in talking therapy implies a deeper understanding that there is more to the interaction than the words used, and that intrapersonal, affective, spiritual and interpersonal constructs (for example) may be involved. The clinicians frequently spoke about shifting their focus onto the patient's world and the patient's role in CBT. Thus, without overtly addressing this with the patient, it seemed that part of the process of CBT was empowering the patient, as Joseph mentions.

'It helps the patient to understand themselves better, if you manage to deliver properly then you give the chance to understand, and understanding and like, giving them the, the chance of finding the solution for themselves. So ... so this is how CBT is useful.' (Joseph)

Different approaches to talking therapy view the locus of control of the intervention in different ways. In CBT a collaborative approach is directly encouraged, so that many aspects of the process 
are shared, from achieving an understanding of the patient's world, to agreeing on which topics are discussed, to planning behavioural change. The clinicians seemed particularly aware of this concept, and described how they collaborated with the patient in order to use CBT, which seemed different from how they might usually enter into a relationship with the patient. Tanzanian society is often understood within a system of hierarchy, meaning patients usually look up to whomever they are seeking help or treatment from for instruction, and clinicians see themselves in the role of directing treatment. This process is significantly different in CBT, as patient and clinician are given active roles. Here, Julius and Pamela refer to this:

'I'm not leading the, the, the session alone, but I'm trying to, to help the patient. Also the patient become his or her own therapist.' (Pamela)

'What I understand is that CBT is about err trying to help people with their problems, especially anxiety, depression, drinking and drug problems, to change their behaviour through participatory methods.' (Julius)

Another sub-theme that emerged was that of 'communication' and given the tendency in Tanzania to view mental illness within a medical model and the clinician-patient interaction within a strict hierarchy, this sub-theme was striking. Interviewees highlighted the importance of both clinician and patient asking questions, giving explanations, and listening to each other. In fact, listening to the patient was repeated over and over by all clinicians, as seen in Amani's account:

'Now we know what is CBT and I can explain to my fellow about CBT. You have to listen to the patient, as I told you. And you have to make a follow-up to listen carefully a patient without any medication.' (Amani)

This is not a unique feature of CBT within the literature, but most certainly is a basic principle of psychotherapy. It seems that listening itself was being labelled by the clinicians in our sample as an active ingredient of the therapy, as reflected in Fred's description of work with one patient, in which he stresses the importance of listening and allowing the patient to speak:

'What really helps a patient is the fact that a client come to acknowledge that there are people who can sit with me and listen to what I have to say, not I listening to what they have to say. So that kind of relationship that you, you build in the sessions, err, it make a client become more calm, more open to his problem or his situation in life and that really he speaks his heart, he gives you everything that was troubling him.' (Fred)

Interestingly, there were several references to communication in practical terms also (important if we are considering the efficacy of using talking therapies in potentially challenging environments). Thus, Pamela noted that she communicated with her patients on the wards as part of the CBT clinic, and also by mobile 'phone when conducting follow-ups.

Although the clinicians referred to the process within the therapist-patient relationship, they also mentioned 'the wider context' meaning other relationships and society, and so this was highlighted as another sub-theme. Many African societies are thought to have a greater focus on social groups and interpersonal relationships rather than on a society constructed of individuals, as many European societies might be described. The clinicians were very aware of how hierarchical relationships and other contextual aspects of Tanzanian life (e.g. religion, employment) could impact on their use of CBT, and prove to be both advantageous and detrimental to the 
therapy. Here, Julius and Gervas give their thoughts on including a wider contextual focus in using CBT with a patient:

'I would have liked to get the, to interview the friends who were close with her, possibly the boyfriend, who was here, and the dean of students.' (Julius)

'So the father, as if he is the one who is sick, does not give chance to the, to the son to talk, yes. Made me in fact feel not pleased.' (Gervas)

Hence, learning skills from systemic approaches to psychotherapy (family therapies) could be particularly important for CBT therapists in Tanzania. It is interesting to note that Gervas himself became upset by the experience he describes, and therefore the therapist's part in the hierarchical structure of those around the patient may be an additional aspect for consideration in CBT training.

Other contextual issues were mentioned, including religious beliefs and socioeconomic problems. The clinicians' encounters with these were evident in their narratives, with comments indicating that they felt CBT was capable of covering an array of issues pertinent to Tanzanians:

'Also the religion itself encourages people to believe in genies and possessions. Maybe those are the factors to try to accommodate in Tanzania, because almost half of the population is Muslim.' (Julius)

'Others could have been even dismissed from work, but because the, the, we can say that they are on treatment they have preserved them, they didn't dismiss them and with the CBT they, they manage to develop insight.' (Pamela)

Although clinicians were aware of these issues, it was not clear whether they felt confident about how they might incorporate this into a CBT formulation with sensitivity, or which specific techniques to employ. In Julius's example, we would hope that a Socratic dialogue to identify the client's Negative Automatic Thoughts and other cognitions about genies would occur, followed by appropriate cognitive challenging and behavioural experiments. We imagine this process could be facilitated by working alongside religious leaders, as has been suggested by Beck (2016) on working with different cultural groups.

\section{Therapist effects}

In discussing their experiences of learning and using CBT, clinicians also spoke about effects they had encountered themselves, revealing another important major theme of 'Therapist effects'. This is notable as the interview did not raise this directly and the interviewees all reported these issues spontaneously. For example, some discussed growing self-awareness regarding how personal beliefs may have influenced their behaviour in clinical situations and external situations in their own lives:

'So when you are testing with your patient even yourself as the therapist, err, you get ideas of, "Ah, maybe I was wrong in this situation", so it is also a self-helping therapy. You are helping your client, yet some of the things that your client tells you seems to be of help to yourself.' (Fred)

There were other accounts from clinicians that described more direct application of CBT to their own personal lives. Violet in particular was able to articulate how learning about the use of CBT for patients had then led her to use it for her own personal stress management:

'I had some high blood pressure sometimes. You remember it? As that ... that was due to tension of work. And I didn't knew what is causing me to have pressure, blood pressure, 
raised blood pressure. But from CBT err, I'm glad to say, to, to, to know that now my pressure is ... [gestures down] ... \{clinician and interviewer talk about blood pressure measurement So no tension, it's ... yes, from CBT training.' (Violet)

Another important sub-theme was that of 'self-disclosure'. All the clinicians described instances of self-disclosure within their use of CBT. Therapist self-disclosure is thought to have both facilitative and hindering effects with respect to the therapeutic relationship in the West (Audet et al., 2010; Jeffrey and Austin, 2007; Kottsieper, 2009). However, certain sub-genres of CBT, such as schema-focused therapy, delineate the value of self-disclosure within therapy. It seems that the clinicians perceived the boundary between themselves (as therapist) and the patient less clearly compared with clinicians in the West. Thus the experience of one person is more readily shared with another, as Pamela describes below:

'Another thing is to, to share a bit about your personal experience with the patient, although not ... with limitations, yeah, you can share so that the patient can find also part and parcel of the, of the treatment, by helping the patient to have learning experience, even from you.' (Pamela)

Self-disclosure was not discussed as a CBT strategy, but may illustrate a Tanzanian adaptation of CBT. 'Storytelling' (in Swahili 'kupiga stori') is thought of as a popular pastime, and clinicians may have unconsciously lapsed into this activity in an effort to provide stimulation for cognitive challenging.

Fred is more open about the blurring of boundaries, which is interesting given Tanzanian individuals' awareness of the hierarchical boundaries between people. He refers to CBT enhancing relationships, even outside of the professional setting.

'CBT tends to gather people around err, you might get friends in there, yeah. So it's much better. People will appreciate more if you see more of them ... \{Interviewer tries to clarify what the clinician means\} ... Well, at work it's mostly professional, but after work, yeah, you'll find people who will want to welcome you at some outing, say you have been helpful, "Can you come, let's share", it's social.' (Fred)

The ethics of blurred boundaries may be questioned by Western clinicians, and this may be a unique feature related to the strongly sociable, albeit structured, nature of Tanzanian society. Whilst self-disclosure could cement power differentials and contradict Socratic approaches, it may also reduce boundaries between clinician and client. This latter point emerged particularly in Fred's comments about broadening their own as well as the client's social network rather than simply being categorized as a professional.

The two sub-themes of 'understanding the model' and 'beginner status' related to the effects of the training on our therapists as professionals. All clinicians were able to outline their understanding of the model, and it appeared had quickly taken up phrases and labels of CBT concepts which they repeated in their narratives. Despite this, clinicians still felt they had beginner status, and lacked confidence in their overall use of CBT. The learning curve for novice therapists of CBT is acknowledged to be steep (Bennett-Levy et al., 2003). However, the clinicians' descriptions seemed in keeping with a natural process of learning, whereby the student starts with a conscious application of skills, only using these new concepts more flexibly with less conscious effort over time. Our clinicians appeared to expect this learning curve and reinforced their new skills and expertise with practice, as Violet describes:

'Sure, I'm not competent on how to go with it, but maybe the time was too short. But maybe slowly, by doing practical and, if God wishes, maybe if could get more training on how to go 
with it, I, I hope I could manage better. But now I'm doing what I do but maybe some, I'm lacking some key skills ... but when I see my clients are getting better I feel happy.' (Violet)

\section{Practicalities}

The final theme in the present study is related to 'Practicalities'. Various practical issues were raised by the interviewed clinicians, as might be expected by any staff working in a resource-poor environment. However, issues related to time and the physical environment in particular recurred as sub-themes. It seemed that previous treatments provided to patients were either in response to acute issues or otherwise spontaneous and unplanned. This meant the structured, planned delivery of CBT over time was new and salient. Joseph and Violet describe the challenges in understaffed environments:

'But because of the environment that we are working, we don't have enough time to deliver, so if you want to do it properly you need time, sometimes we run short of it.' (Joseph)

'It is time consumer, you need more time. You don't try the job may be ten minutes and finish and another. No! It needs time, may be even one hour ...' (Violet)

This may indeed be a practical issue of not enough staff, but it may also be related to managerial issues or even personal professional practice, with staff practising CBT having to arrange the time they have available to do their clinical work in a new way. Julius phrases this succinctly:

'I think particularly here, it's just the time, the time factor. I have to steal time to do it.' (Julius)

The clinicians also highlighted how 'facilities' (usually referring to poor environmental resources) affected them. For example, Julius mentions his view that in order to carry out CBT effectively with patients, a quiet, enclosed environment without interruptions and dedicated to CBT is needed:

'It's difficult, because of the environment. And err the lack of conducive environment in which to conduct the, the, the clinics. Place, the quiet place where there is no interference because you know you've seen my office, there every 30 minutes there will be a knock. So we need may if we had a clinic separately.' (Julius)

Such an area dedicated to a CBT clinic was alluded to by others also, such as Gervas, who highlighted the need for other materials such as literature. As CBT is based on evidence, it goes without saying that clinicians require such resources for themselves and their clients in order to use CBT effectively and ethically. However, even with these restraints, the overall experience of using CBT at Mirembe Hospital was positive; as Fred states 'Well, CBT is exciting', and Violet says 'I'm proud to know CBT'. A final quote from Julius illustrates initial signs that both clinicians and clients had this positive experience:

'I do gain confidence with the time and the patients gain confidence, you know, they know my number and they try to, actually they tend among themselves to call, "Go to someone, go to this one".' (Julius)

\section{Discussion}

Within this study, five superordinate themes emerged, and these interlinked themes highlighted how CBT was different from previous therapeutic techniques used at Mirembe Hospital (dominated by a medical model). The participants described how this novel technique lacked 
awareness within the community and would require further training of professionals and a shift in attitudes of service users to become more acceptable. The process of CBT offered empowerment to the patients in the form of collaboration with the hospital staff. This increased communication between professionals and patients engendered greater contact and a more in-depth understanding of the patients' personal context (such as family life) which can assist the therapeutic process and outcomes. The communication skills which clinicians felt they had gained are not necessarily specific to CBT, although they would certainly have that specific flavour (e.g. guided discovery), but it is of course possible that training Tanzanian mental health staff in any of the talking therapies would enhance communication skills, with a general positive effect (Keijsers et al., 2000). We note that the clinicians were all experienced in dealing with patients with mental health problems, but CBT offered not only a new theory and set of techniques to use, but a new way of developing a therapeutic relationship with the patient. It seems many of the staff trained had a positive experience delivering CBT, but further to that also benefited personally. This is consistent with other literature exploring CBT training for clinicians in non-Western settings (e.g. Bennett-Levy et al., 2014). A further notable finding was how the participants in this study used self-disclosure more than would potentially be seen in a Western session (although we know of no studies that have specifically asked UK therapists about their use of self-disclosure). All the clinicians exhibited an understanding of the CBT model even though they felt themselves to be beginners in this new field. Finally, in terms of practicalities, time factors and facilities were believed to have an impact upon the delivery of CBT. Notably, in spite of this, all of the participants stated that they felt positive about the use of this new tool and would like to continue its use in future.

With respect to learning processes of students of $\mathrm{CBT}$, further practice in self-reflection and other CBT techniques such as 'self-practice' are thought to enhance new skill accumulation (Bennett-Levy et al., 2003); therefore we would recommend this as an essential part of continuing training and supervision to those completing initial courses in CBT. Whilst there was opportunity for reflection and practice of CBT skills during the training, this was not a core part of the course. The process of interviewing the clinicians may have allowed them some time for reflection. Importantly, in a study looking at training Aboriginal counsellors in CBT, those using self-practice spontaneously, as in our group, showed increased confidence and competence as therapists (Bennett-Levy et al., 2015).

We acknowledge that this study is a very preliminary step in investigating how CBT may contribute to mental health services in Tanzania. Having introduced a relatively short training course to a small group of clinicians working in typical circumstances in mental health services in Tanzania, we have evidence that the training and basic model is acceptable (see also an evaluation of the pilot training course in Stone and Warren, 2011). The next steps would be to make cultural modifications and conduct a study looking at effectiveness and acceptability of 'Tanzanian CBT' in a specified patient cohort. We are not aware of any plans to develop these modifications and evaluate them as this would require a significant investment in human resources, time and funding. However, it is worth sharing with the readership that there is now a Tanzanian permanent member of staff at Mirembe who practises CBT and offers CBT consultation to others.

It should be noted that although cultural adaptation of psychological therapies seems necessary and a natural consequence of the reality of introducing concepts originally developed elsewhere, without validation, adaptations may potentially lead to sub-optimal effectiveness and efficacy. In looking at adapting and validating instruments to assess mental health symptoms, Kohrt et al. (2011) compared transcultural translation and an adapted validation procedure. By proposing six questions to analyse adaptation procedures of mental health interventions, they propose that different approaches will fit different settings and cultures. We would argue that gaining the views of those using CBT in Tanzania is part of such adapted validation procedures; gaining these views allows greater confidence in answering concerns about which constructs CBT is addressing and what idioms and structures are more or less helpful in the Tanzanian context. 
Our study offers some insights into how CBT might be adapted for the Tanzanian context. For example, unexpected comments relating to self-disclosure and the effects of therapy not only on the patient but also the wider society imply CBT has potential in the development of Tanzanian society, especially with respect to supporting individuals in terms of their mental health, which has the ability to empower people to work. Usually overseas development programmes focus on political and socio-economic issues, but neglect psychological factors of development, and some writers have argued for psychological empowerment as key to sustainable economic development (Onyishi and Agbo, 2010). The World Health Organization (WHO) recognizes that the dearth of mental health support and services is leading to a global human rights crisis. Thirty per cent of countries lack an exclusive budget for mental health care, and of those that do set aside funds, $20 \%$ allocate less than $1 \%$ of their total health budget to mental health (World Health Organization, 2001). Adequate funding is certainly required to reduce the impact of mental health issues in low-income countries; CBT is a cost-effective way to treat mental health conditions (Wiles et al., 2016)

Controversy is often generated by studies such as this and we need to acknowledge the debates concerning the validity of using Western frameworks (including CBT) to generate universal paradigms in mental health. Summerfield (2008) is particularly clear in his challenge that numerous aspects of mental disorders vary greatly between cultures, meaning social conditions and local concepts of both 'mental' and 'health' need to be understood and he argues for qualitative research into mental health in non-Western settings. Given the scant literature on this subject, we strongly support this recommendation. Preliminary exploration of patients', carers' and health professionals' views seems a necessary first step in understanding explanatory models of mental health and illness in a particular country and culture, which then allows adaptation of CBT models; this has been done for psychosis and depression in Pakistan (Naeem et al., 2011, 2016). Nevertheless we would argue that this is not a reason against investigating how a known effective talking treatment may be applied in a new cultural setting (Tanzania). As long as this is done appropriately, with the consent of and in partnership with local people, as we hope we have done, we believe there is an ethical argument for such research (Singer et al., 2015).

We are aware that our study has limitations, which may affect the validity of its findings. Firstly, we had a relatively low number of interviewees. The response rate was seven out of the 19 trainees and the main reason for this was that several of the trainees did not live locally (in fact, several hundreds of miles away) and so it was not practically possible for them to contribute to any face-to-face follow-up work. Therefore we might view the response rate more pragmatically as seven out of 12 (58.3\%). Furthermore, guidance about sample size when using an IPA methodology states 'There is no right answer to the question of sample size. It partly depends on several factors: the degree of commitment to the case study level of analysis and reporting, the richness of the individual cases, and the constraints one is operating under ... In the recent past, five or six has been recommended as a decent sample size ...' (p. 56, Smith and Osborn, 2007). Moreover, we believe our study demonstrated a wealth of material and the themes identified were formed across all participants' interviews with sufficient saturation (as per guidance from Smith et al., 2007).

We need to explicitly acknowledge that our findings are specific to the group of clinicians who undertook the CBT training in Mirembe hospital; thus it is important to acknowledge that we cannot simply generalize the findings of this study to a wider population. The training and supervision provided was specific as taught at one particular time by one of the authors, i.e. it was not and could not be an extensive, externally verified or validated course. We are mindful that the findings do not say anything about the views of clients, nor the effectiveness or acceptability of the therapy to them. We are also aware, that as the interviews were not conducted in Swahili, the English is sometimes clearly 'broken' and it could therefore be argued that comments and concepts could not actually be communicated in a valid way. However, as most teaching is carried out in English in Tanzania, we would respond that this would have only a minor effect. 
Importantly, as the trainer also conducted the interviews it may have been very difficult for clinicians to express any negative views, and perhaps more so given the prevailing culture in Tanzania being one that respects professionals, teachers and Europeans and also can sacrifice truth for social soothing. Thus, the positive views could be challenged as being a positive 'spin' in order not to challenge, or seem rude or ungrateful; we hope that, given that the interviewees were able to discuss in detail their views, it is unlikely this is wholly the case. However, some aspects of this remain highly probable. The trainer did not evaluate the participants in any way (formal or informal) with respect to their work role and, as interviewer, emphasized the interest that their honest views would provoke, thereby hoping to diminish any power differentials, which clearly could have been present.

These issues, together with the low number of interviewees, mean our research could be easily dismissed as biased and methodologically flawed. Whilst we understand this opinion, we feel very strongly that in contexts where research is carried out in difficult circumstances such as in developing countries, where there is little support from large research grants (this research was prompted and supported purely by the trainer, co-author and those involved in the training, i.e. Tanzania trainees and other mental health staff at Mirembe) and where groups are struggling to implement new ideas in much-needed fields, more understanding is required with an acceptance that limitations and obstacles are a disagreeable but an unavoidable part of many pilot studies in LAMIC.

Acknowledgements. We would like to acknowledge and thank all those who supported this study being carried out from Mirembe Hospital, the Tanzanian Ministry of Health and Skillshare International. Particular thanks go to Dr Joseph Mbatia and Dr Erasmus Mndeme who gave valuable feedback on the CBT training itself as well as writing of the paper. Most importantly, we would like to thank all clinicians who participated and were interviewed. We appreciate their dedication and enthusiasm throughout.

Financial support. This research received no specific grant from any funding agency, commercial or not-for-profit sectors.

Conflicts of interest. Lydia Stone and Megan Arroll have no conflicts of interest with respect to this publication.

Ethics statement. We have at all times worked to abide by the Ethical Principles of Psychologists and Code of Conduct as set out by the APA, even though formal ethical approval was not needed or asked for by the organizations in which the research was undertaken.

Key practice points.

(1) Relatively brief CBT training to clinicians from (and working in) LAMIC such as Tanzania appears to hold promise in its ability to communicate some of the core tenets of CBT practice, such as empowering the client, the importance of therapeutic alliance and transparency in how the therapy works.

(2) Using a qualitative approach allowed the identification of a number of characteristics in practice that could help the development of culturally sensitive CBT, specific to Tanzania - for example, the use of self-disclosure, story-telling and including family members.

(3) Despite the small scale of this study and other limitations, our findings have shown that generating themes using a qualitative approach is a useful first step in considering factors pertinent to the teaching and practice of CBT in a developing country such as Tanzania.

\section{Further reading}

Naeem, F. and Kingdon, D. G. (eds) (2012). CBT in Non-Western Cultures. Psychology Research Progress: Focus Civilizations and Cultures.

Naeem, F., Gobbi, M., Ayub, M. and Kingdon, D. (2010). Psychologists experience of cognitive behaviour therapy in a developing country: a qualitative study from Pakistan. International Journal of Mental Health Systems, 4, 2.

Ngoma, M. C., Prince, M. and Mann, A. (2003). Common mental disorders among those attending primary health clinics and traditional healers in urban Tanzania. The British Journal of Psychiatry, 183, 349-355. 


\section{References}

Aina, O. (2006). 'Psychotherapy by environmental manipulation' and the observed symbolic rites on prayer mountains in Nigeria. Mental Health, Religion and Culture, 9, 1-13.

Audet, C. T., \& Everall, R. D. (2010). Therapist self-disclosure and the therapeutic relationship: a phenomenological study from the client perspective. British Journal of Guidance \& Counselling, 38, 327-342.

Beck, A. (2016). Transcultural Cognitive Behaviour Therapy for Anxiety and Depression: A Practical Guide. Abingdon, UK and New York, USA: Routledge.

Bennett-Levy, J., Lee, N., Travers, K., Pohlman, S. and Hamernik, E. (2003). Cognitive therapy from the inside: enhancing therapist skills through practising what we preach. Behavioural and Cognitive Psychotherapy, 31, 143-158.

Bennett-Levy, J., Wilson, S., Nelson, J., Rotumah, D., Ryan, K., Budden, W. and Stirling, J. (2015). Spontaneous selfpractice of CBT by Aboriginal counsellors during and following CBT training: a retrospective analysis of facilitating conditions and impact. Australian Psychologist, 50, 329-334.

Bennett-Levy, J., Wilson, S., Nelson, J., Stirling, J., Ryan, K., Rotumah, D. and Beale, D. (2014). Can CBT be effective for Aboriginal Australians? Perspectives of Aboriginal practitioners trained in CBT. Australian Psychologist, 49, 1-7.

Bolton, P. and Wilk, C. M. (2004). How do Africans view the impact of HIV? A report from a Ugandan community. AIDS Care, 16, 123-128.

Chowdhary, N., Sikander, S., Atif, N., Singh, N., Ahmad, I., Fuhr, D. C. and Patel, V. (2014). The content and delivery of psychological interventions for perinatal depression by non-specialist health workers in low and middle income countries: a systematic review. Best Practice and Research Clinical Obstetrics and Gynaecology, 28, 113-133.

Grazebrook, K. and Garland, A. (2005). What are cognitive and/or behavioural psychotherapies? British Association for Behavioural and Cognitive Psychotherapies. Retrieved from: http://www.babcp.com/babcp/what_is_CBT.htm (accessed 21 July 2009).

Guba, E. G. and Lincoln, Y. S. (1981). Effective Evaluation: Improving the Usefulness of Evaluation Results through Responsive and Naturalistic Approaches. USA: Jossey-Bass.

Jeffrey, A., \& Austin, T. (2007). Perspectives and practices of clinician self-disclosure to clients: a pilot comparison study of two disciplines. American Journal of Family Therapy, 35, 95-108.

Jones, D. L., Ross, D., Weiss, S. M., Bhat, G. and Chitalu, N. (2005). Influence of partner participation on sexual risk behavior reduction among HIV-positive Zambian women. Journal of Urban Health, 82, iv92-iv100.

Keijsers, G. P. J., Schaap, C. P. D. R. and Hoogduin, C. A. L. (2000). The impact of interpersonal patient and therapist behavior on outcome in cognitive-behavior therapy: a review of empirical studies. Behavior Modification, 24, $264-297$.

Kohrt, B. A., Jordans, M. J., Tol, W. A., Luitel, N. P., Maharjan, S. M. and Upadhaya, N. (2011). Validation of crosscultural child mental health and psychosocial research instruments: adapting the Depression Self-Rating Scale and Child PTSD Symptom Scale in Nepal. BMC Psychiatry, 11, 127.

Kottsieper, P. (2009). Experiential knowledge of serious mental health problems: one clinician and academic's perspective. Journal of Humanistic Psychology, 49, 174-192.

Marwick, K. F. and Kaaya, S. F. (2010). Prevalence of depression and anxiety disorders in HIV-positive outpatients in rural Tanzania. AIDS Care, 22, 415-419.

Mbatia, J. and Jenkins, R. (2010). Development of a mental health policy and system in Tanzania: an integrated approach to achieve equity. Psychiatric Services, 61, 1028-1031.

Naeem, F., Gobbi, M., Ayub, M. and Kingdon, D. (2010). Psychologists experience of cognitive behaviour therapy in a developing country: a qualitative study from Pakistan. International Journal of Mental Health Systems, 4, 2.

Naeem, F., Habib, N., Gul, M., Khalid, M., Saeed, S., Farooq, S. and Kingdon, D. (2016). A qualitative study to explore patients', carers' and health professionals' views to culturally adapt CBT for psychosis (CBTp) in Pakistan. Behavioural and Cognitive Psychotherapy, 44, 43-55. doi: 10.1017/S1352465814000332

Naeem, F., Waheed, W., Gobbi, M., Ayub, M. and Kingdon, D. (2011). Preliminary evaluation of culturally sensitive CBT for depression in Pakistan: findings from Developing Culturally sensitive CBT Project (DCCP). Behavioural and Cognitive Psychotherapy, 39, 165-173. doi: 10.1017/S1352465810000822

Ngoma, M. C., Prince, M. and Mann, A. (2003). Common mental disorders among those attending primary health clinics and traditional healers in urban Tanzania. The British Journal of Psychiatry, 183, 349-355.

Onyishi, I. E. and Agbo, A. (2010). Psychological empowerment and development of entrepreneurship women: implications for sustainable economic development in Nigeria. Gender and Behaviour, 8, 3048-3067.

Osinowo, H., Olley, B. and Adejumo, A. (2004). Evaluation of the effect of cognitive therapy on perioperative anxiety and depression among Nigerian surgical patients. West African Journal of Medicine, 22, 338-342.

Papas, R. K., Sidle, J. E., Martino, S., Baliddawa, J. B., Songole, R., Omolo, O. E. and Nafula, T. (2010). Systematic cultural adaptation of cognitive-behavioral therapy to reduce alcohol use among HIV-infected outpatients in western Kenya. AIDS and Behavior, 14, 669-678.

Patel, V., Araya, R., Chatterjee, S., Chisholm, D., Cohen, A., De Silva, M. and Van Ommeren, M. (2007). Global Mental Health 3: treatment and prevention of mental disorders in low-income and middle-income countries. Lancet, 370, 991-1005. 
Patel, V., Chowdhary, N., Rahman, A. and Verdeli, H. (2011). Improving access to psychological treatments: lessons from developing countries. Behaviour Research and Therapy, 49, 523-528.

Roth, A. and Fonagy, P. (2005). What Works for Whom?: A Critical Review of Psychotherapy Research. New York, USA: Guilford Press.

Saxena, S., Thornicroft, G., Knapp, M. and Whiteford, H. (2007). Global Mental Health 2 - resources for mental health: scarcity, inequity, and inefficiency. Lancet, 370, 878-890.

Semrau, M., Evans-lacko, S., Alem, A., Ayuso-mateos, J. L., Chisholm, D., Gureje, O., Hanlon, C., Jordans, M., Kigozi, F., Lempp, H., Lund, C., Petersen, I., Shidhaye, R., \& Thornicroft, G. (2015). Strengthening mental health systems in lowand middle-income countries: the Emerald programme. BMC Medicine, 13, 79.

Singer, J., Bennett-Levy, J. and Rotumah, D. (2015). 'You didn't just consult community, you involved us': transformation of a 'top-down'Aboriginal mental health project into a 'bottom-up'community-driven process. Australasian Psychiatry, 23, 614-619.

Smith, J. A. (2011). Evaluating the contribution of interpretative phenomenological analysis: a reply to the commentaries and further development of criteria. Health Psychology Review, 5, 55-61.

Smith, J. A., Larkin, M. and Flowers, P. (2009). Interpretative Phenomenological Analysis: Theory, Method and Research. Sage Publications Limited.

Smith, J. A., \& Osborn, M. (2003). Chapter 4: Interpretive phenomenological analysis. In J. Smith (ed), Qualitative Psychology: A Practical Guide to Methods, pp. 53-80. London, UK: Sage Publications.

Smith, J. A. and Osborn, M. (2007). Interpretative phenomenological analysis. In JA Smith (ed), Qualitative Psychology: A Practical Guide to Research Methods. London, UK: Sage Publications.

Stewart, D. W., Shamdasani, P. N. and Rook, D. W. (2007) Focus Groups - Theory and Practice, 2nd edn. Applied Social Research Methods Series, vol. 20. New Delhi: Sage Publications.

Stone, L. and Warren, F. (2011). Cognitive behaviour therapy training in a developing country: a pilot study in Tanzania. The Cognitive Behaviour Therapist, 4, 139-151.

Summerfield, D. (2008). How scientifically valid is the knowledge base of global mental health? British Medical Journal, 336, 992.

Thornicroft, G., \& Semrau, M. (2018). Mental health capacity building in low and middle income countries: the Emerald Programme. Epidemiology and Psychiatric Sciences, 27, 1-2.

Wiles, N. J., Thomas, L., Turner, N., Garfield, K., Kounali, D., Campbell, J. and Williams, C. (2016). Long-term effectiveness and cost-effectiveness of cognitive behavioural therapy as an adjunct to pharmacotherapy for treatmentresistant depression in primary care: follow-up of the CoBalT randomised controlled trial. The Lancet Psychiatry, 3, 137-144.

World Health Organization (2001). The World Health Report 2001: Mental Health: New Understanding, New Hope. Geneva: World Health Organization.

World Health Organization (WHO) (2017). mhGAP FORUM 2017.

Cite this article: Stone L and Arroll MA. Cognitive behavioural therapy training in Tanzania: a qualitative study of clinicians' experiences. The Cognitive Behaviour Therapist. https://doi.org/10.1017/S1754470X19000084 